Acta Crystallographica Section E

Structure Reports

Online

ISSN 1600-5368

\section{2-\{[4-(Pyridin-2-yl)pyrimidin-2-yl]- sulfanyl\}acetic acid}

\section{Lin Wang and Hua-Ze Dong*}

Deparment of Chemistry and Chemical Engineering, Hefei Normal University, Hefei 230061, People's Republic of China

Correspondence e-mail: dapdong@163.com

Received 17 August 2011; accepted 28 September 2011

Key indicators: single-crystal X-ray study; $T=291 \mathrm{~K}$; mean $\sigma(\mathrm{C}-\mathrm{C})=0.002 \AA$; $R$ factor $=0.035 ; w R$ factor $=0.096 ;$ data-to-parameter ratio $=16.3$.

In the title molecule, $\mathrm{C}_{11} \mathrm{H}_{9} \mathrm{~N}_{3} \mathrm{O}_{2} \mathrm{~S}$, the pyridine and pyrimidine rings are almost parallel [dihedral angle $=6.7(1)^{\circ}$ ]. In the crystal, adjacent molecules are joined by $\mathrm{O}-\mathrm{H} \cdots \mathrm{N}$ and $\mathrm{C}-$ $\mathrm{H}$... O hydrogen bonds, leading to the formation of a sheet parallel to $(10 \overline{2})$.

\section{Related literature}

For details of the synthesis and general background, see: Dong et al. (2009); Wang (2011). For the crystal structures of coordination complexes with related ligands, see: Du et al. (2004); Zhu et al. (2009).

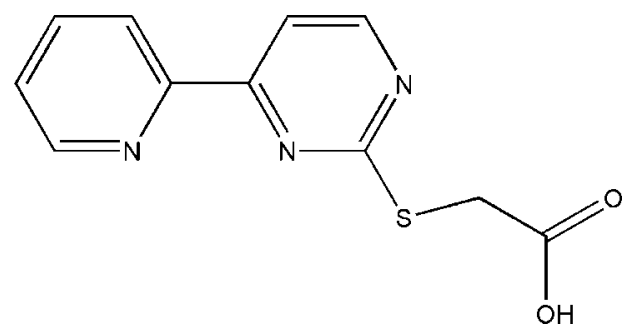

\section{Experimental}

Crystal data

$\mathrm{C}_{11} \mathrm{H}_{9} \mathrm{~N}_{3} \mathrm{O}_{2} \mathrm{~S}$

$M_{r}=247.28$

Monoclinic, $P 2_{1} / c$

$a=6.5722(2) \AA$
$Z=4$

Mo $K \alpha$ radiation

$\mu=0.29 \mathrm{~mm}^{-1}$

Data collection

Bruker SMART CCD area-detector diffractometer

Absorption correction: multi-scan (SADABS; Bruker, 2000)

$T_{\min }=0.920, T_{\max }=0.950$

Refinement

$R\left[F^{2}>2 \sigma\left(F^{2}\right)\right]=0.035$

$w R\left(F^{2}\right)=0.096$

$S=1.05$

2524 reflections
$T=291 \mathrm{~K}$
$0.28 \times 0.20 \times 0.18 \mathrm{~mm}$

10868 measured reflections 2524 independent reflections 2116 reflections with $I>2 \sigma(I)$ $R_{\text {int }}=0.022$

155 parameters

$\mathrm{H}$-atom parameters constrained $\Delta \rho_{\max }=0.21$ e $\AA^{-3}$ $\Delta \rho_{\min }=-0.24$ e $\AA^{-3}$

Table 1

Hydrogen-bond geometry $\left(\AA{ }^{\circ}\right)$.

\begin{tabular}{|c|c|c|c|c|}
\hline$D-\mathrm{H} \cdots A$ & $D-\mathrm{H}$ & $\mathrm{H} \cdots A$ & $D \cdots A$ & $D-\mathrm{H} \cdots A$ \\
\hline $\mathrm{O} 2-\mathrm{H} 2 \cdots \mathrm{N} 2^{\mathrm{i}}$ & 0.82 & 1.87 & $2.694(2)$ & 178 \\
\hline $\mathrm{C} 2-\mathrm{H} 2 A \cdots \mathrm{O} 1^{\mathrm{ii}}$ & 0.93 & 2.58 & $3.230(2)$ & 127 \\
\hline $\mathrm{C} 8-\mathrm{H} 8 \cdots \mathrm{O} 2^{\mathrm{iii}}$ & 0.93 & 2.48 & $3.392(2)$ & 165 \\
\hline $\mathrm{C} 9-\mathrm{H} 9 \cdots \mathrm{O}^{\mathrm{iv}}$ & 0.93 & 2.45 & $3.296(2)$ & 151 \\
\hline
\end{tabular}

Data collection: SMART (Bruker, 2000); cell refinement: SAINT (Bruker, 2000); data reduction: $S A I N T$; program(s) used to solve structure: SHELXTL (Sheldrick, 2008); program(s) used to refine structure: SHELXTL; molecular graphics: SHELXTL; software used to prepare material for publication: SHELXTL.

This work was supported by the National Science Foundation of China (No. 20871039), the Program for Excellent Young Talents in Universities of Anhui Province (2011SQRL128) and the Science Foundation of Hefei Normal University(2010kj01zd).

Supplementary data and figures for this paper are available from the IUCr electronic archives (Reference: KJ2186).

\title{
References
}

Bruker (2000). SADABS, SMART and SAINT. Bruker AXS Inc., Madison, Wisconsin, USA.

Dong, H. Z., Zhao, J., Zhu, H. B. \& Gou, S. H. (2009). Polyhedron, 28, 10401048.

Du, M., Zhao, X. J. \& Wang, Y. (2004). Dalton Trans. pp. 2065-2072.

Sheldrick, G. M. (2008). Acta Cryst. A64, 112-122.

Wang, C.-H. (2011). Acta Cryst. E67, o690.

Zhu, H.-B., Xu, G. \& Sun, Y.-Y. (2009). Acta Cryst. E65, m1126. 


\section{supporting information}

Acta Cryst. (2011). E67, o2916 [doi:10.1107/S1600536811039791]

\section{2-\{[4-(Pyridin-2-yl)pyrimidin-2-yl]sulfanyl\}acetic acid}

\section{Lin Wang and Hua-Ze Dong}

\section{S1. Comment}

The rational dsign and assembly of new coordination polymers with with thioethers derived from 4-pyridinyl pyrimidine-2-thiol have received considerable attention in recent years (Dong et al., 2009; Du et al., 2004; Wang, 2011; Zhu et al., 2009). Here we report the crystal structure of a newly synthesized compound derived from 4-(4pyridinyl)pyrimidine-2-thiol.

The molecular structure of title compound is shown in Fig. 1 together with the atom-numbering scheme. The pyridine and pyrimidine rings are almost parallel with a dihedral angle of $6.7(1)^{\circ}$. Molecules are linked by $\mathrm{O}-\mathrm{H} \cdots \mathrm{N}$ hydrogen bonds into a chain running in direction $\left[\begin{array}{lll}2 & 0 & 1\end{array}\right]$. $\mathrm{C}-\mathrm{H} \cdots \mathrm{O}$ interactions join these chains into a two-dimensional network with base vectors [ $\left[\begin{array}{lll}2 & 0 & 1\end{array}\right]$ and $\left[\begin{array}{lll}0 & 1 & 0\end{array}\right]$ (equivalent to a sheet parallel to the $\left(\begin{array}{lll}1 & 0 & -2\end{array}\right)$ lattice planes). Geometrical details are given in Table 1; a plot is given in Fig. 2.

\section{S2. Experimental}

All solvents and chemicals were of analytical grade and were used without further purification. The title compound was prepared by a similar procedure as reported in the literature (Dong et al., 2009). To a solution of 4-(4pyridinyl)pyrimidine-2-thiol $(3.78 \mathrm{~g}, 20 \mathrm{mmol})$ and sodium hydroxide $(0.80 \mathrm{~g}, 20 \mathrm{mmol})$ in water $(30 \mathrm{ml})$, 2-bromoacetic acid $(2.78 \mathrm{~g}, 20 \mathrm{mmol})$ in water $(30 \mathrm{ml})$ was added. The mixture was stirred at room temperature for $4 \mathrm{~h}$. Dilute hydrochloric acid was added to the reacted solution until the $\mathrm{pH}$ was about 4 . Precipitates were filtered, washed by water and ethanol, and dried in vacuum. Single crystals suitable for X-ray diffraction were grown from a methanol solution by slow evaporation in air at room temperature.

\section{S3. Refinement}

All hydrogen atoms were geometrically positioned $(\mathrm{C}-\mathrm{H} 0.93-0.97 \AA)$ and refined as riding, with $U_{\text {iso }}(\mathrm{H})=1.2-1.5 U_{\text {eq }}$ of the parent atom. 


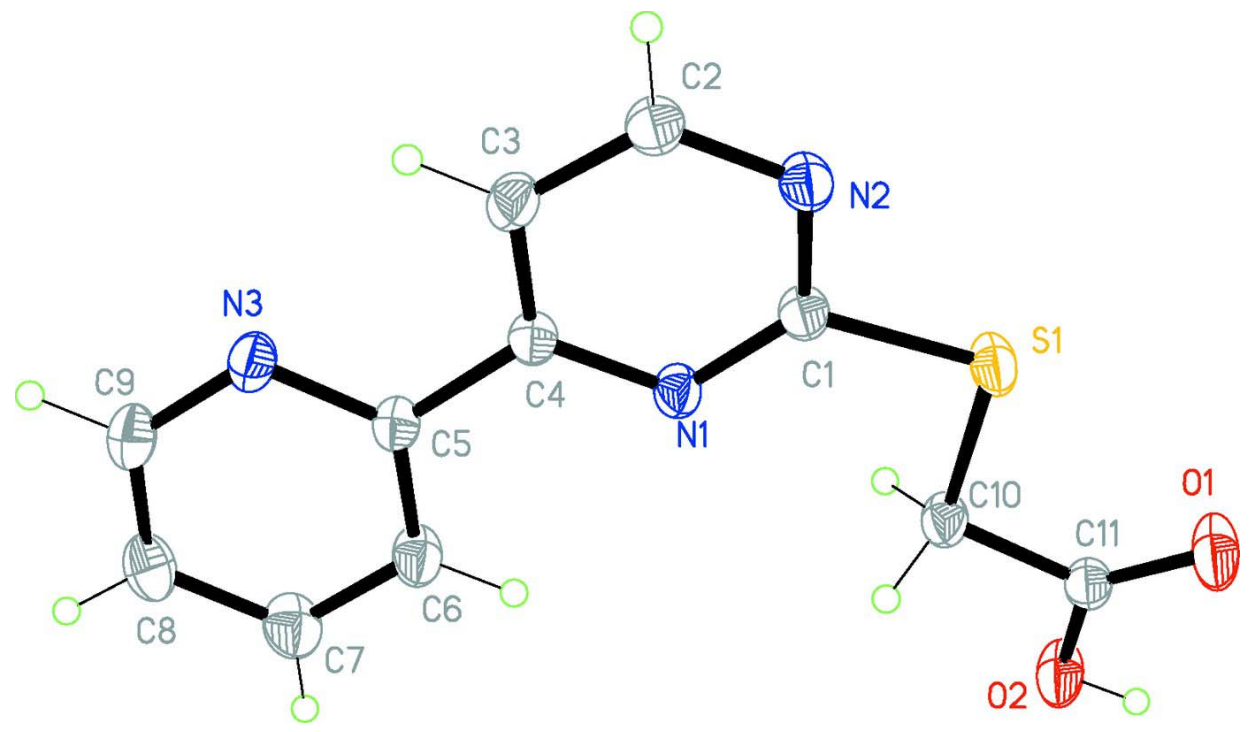

Figure 1

The structure of the title compound, showing $30 \%$ probability displacement ellipsoids.

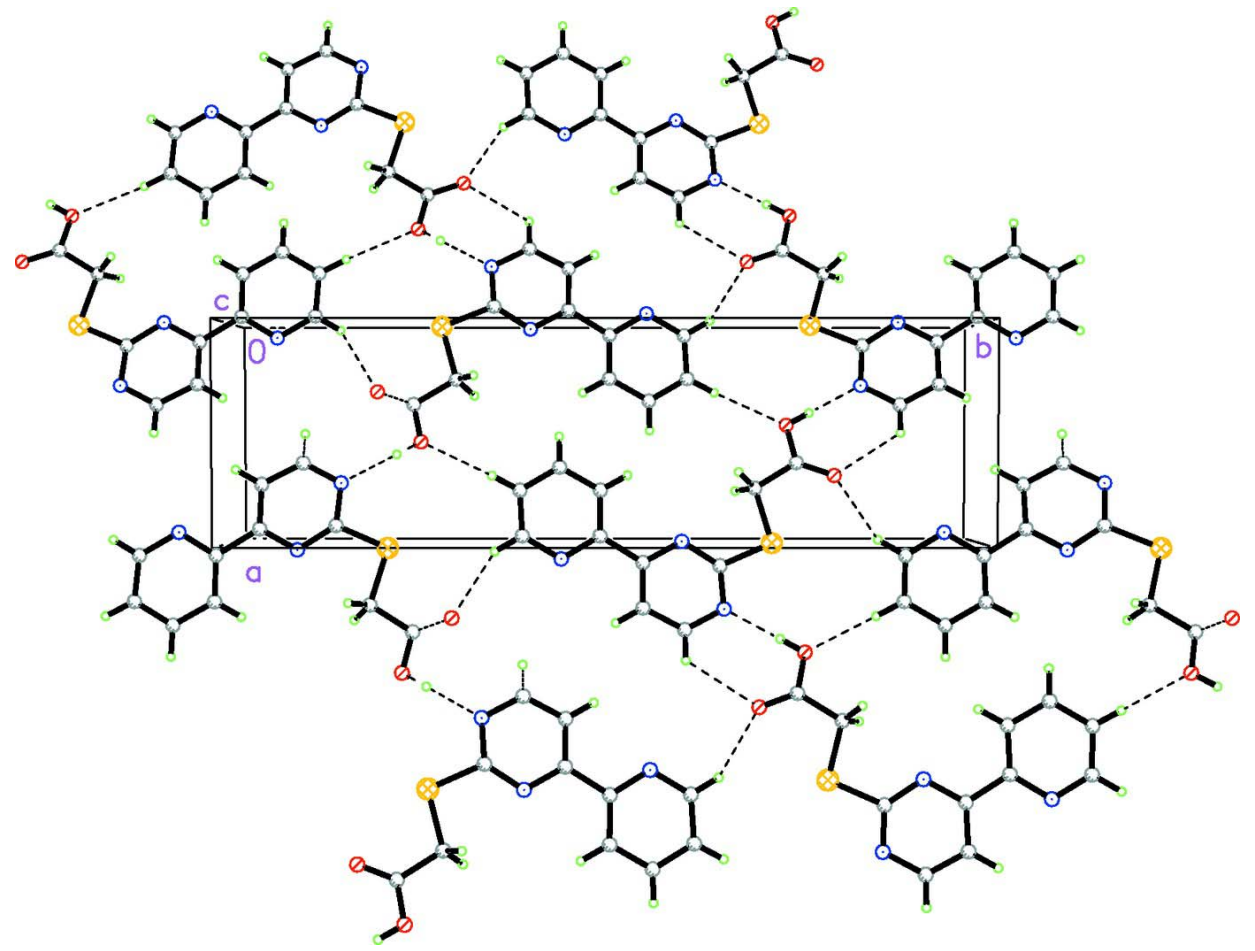

Figure 2

Perspective view of the crystal packing. Hydrogen bonding interactions are indicated with dashed lines.

\section{2-\{[4-(Pyridin-2-yl)pyrimidin-2-yl]sulfanyl\}acetic acid}

Crystal data

$\mathrm{C}_{11} \mathrm{H}_{9} \mathrm{~N}_{3} \mathrm{O}_{2} \mathrm{~S}$

$M_{r}=247.28$

Monoclinic, $P 2_{1} / c$

Hall symbol: -P 2ybc

$a=6.5722(2) \AA$

$b=22.4650(8) \AA$ 
$c=7.4314$ (2) $\AA$

$\beta=93.237(2)^{\circ}$

$V=1095.45(6) \AA^{3}$

$Z=4$

$F(000)=512.0$

$D_{\mathrm{x}}=1.499 \mathrm{Mg} \mathrm{m}^{-3}$

Mo $K \alpha$ radiation, $\lambda=0.71073 \AA$

Data collection

Bruker SMART CCD area-detector diffractometer

Radiation source: fine-focus sealed tube

Graphite monochromator

$\varphi$ and $\omega$ scans

Absorption correction: multi-scan

(SADABS; Bruker, 2000)

$T_{\min }=0.920, T_{\max }=0.950$

Refinement

Refinement on $F^{2}$

Least-squares matrix: full

$R\left[F^{2}>2 \sigma\left(F^{2}\right)\right]=0.035$

$w R\left(F^{2}\right)=0.096$

$S=1.05$

2524 reflections

155 parameters

0 restraints

Primary atom site location: structure-invariant direct methods
Cell parameters from 2524 reflections

$\theta=1.8-27.5^{\circ}$

$\mu=0.29 \mathrm{~mm}^{-1}$

$T=291 \mathrm{~K}$

Block, pale yellow

$0.28 \times 0.20 \times 0.18 \mathrm{~mm}$

10868 measured reflections

2524 independent reflections

2116 reflections with $I>2 \sigma(I)$

$R_{\text {int }}=0.022$

$\theta_{\max }=27.5^{\circ}, \theta_{\min }=1.8^{\circ}$

$h=-8 \rightarrow 8$

$k=-24 \rightarrow 29$

$l=-9 \rightarrow 9$

Secondary atom site location: difference Fourier map

Hydrogen site location: inferred from

neighbouring sites

$\mathrm{H}$-atom parameters constrained

$w=1 /\left[\sigma^{2}\left(F_{0}{ }^{2}\right)+(0.0449 P)^{2}+0.289 P\right]$

where $P=\left(F_{\mathrm{o}}^{2}+2 F_{\mathrm{c}}^{2}\right) / 3$

$(\Delta / \sigma)_{\max }=0.001$

$\Delta \rho_{\max }=0.21 \mathrm{e} \AA^{-3}$

$\Delta \rho_{\min }=-0.24 \mathrm{e} \AA^{-3}$

Special details

Experimental. The structure was solved by direct methods (Bruker, 2000) and successive difference Fourier syntheses.

Geometry. All e.s.d.'s (except the e.s.d. in the dihedral angle between two 1.s. planes) are estimated using the full covariance matrix. The cell e.s.d.'s are taken into account individually in the estimation of e.s.d.'s in distances, angles and torsion angles; correlations between e.s.d.'s in cell parameters are only used when they are defined by crystal symmetry. An approximate (isotropic) treatment of cell e.s.d.'s is used for estimating e.s.d.'s involving 1.s. planes.

Refinement. Refinement of $F^{2}$ against ALL reflections. The weighted $R$-factor $w R$ and goodness of fit $S$ are based on $F^{2}$, conventional $R$-factors $R$ are based on $F$, with $F$ set to zero for negative $F^{2}$. The threshold expression of $F^{2}>\sigma\left(F^{2}\right)$ is used only for calculating $R$-factors(gt) etc. and is not relevant to the choice of reflections for refinement. $R$-factors based on $F^{2}$ are statistically about twice as large as those based on $F$, and $R$ - factors based on ALL data will be even larger.

Fractional atomic coordinates and isotropic or equivalent isotropic displacement parameters $\left(\AA^{2}\right)$

\begin{tabular}{lllll}
\hline & $x$ & $y$ & $z$ & $U_{\text {iso }} * / U_{\text {eq }}$ \\
\hline C1 & $-0.0901(2)$ & $0.34978(6)$ & $0.26132(19)$ & $0.0342(3)$ \\
C2 & $-0.3668(2)$ & $0.39680(7)$ & $0.1300(2)$ & $0.0412(4)$ \\
H2A & -0.4973 & 0.3959 & 0.0747 & $0.049^{*}$ \\
C3 & $-0.2718(2)$ & $0.45062(7)$ & $0.1562(2)$ & $0.0391(3)$ \\
H3 & -0.3370 & 0.4860 & 0.1229 & $0.047^{*}$ \\
C4 & $-0.0745(2)$ & $0.45042(6)$ & $0.23433(19)$ & $0.0327(3)$ \\
C5 & $0.0431(2)$ & $0.50633(6)$ & $0.25998(19)$ & $0.0333(3)$ \\
C6 & $0.2462(2)$ & $0.50591(7)$ & $0.3208(2)$ & $0.0427(4)$ \\
H6 & 0.3120 & 0.4702 & 0.3493 & $0.051^{*}$
\end{tabular}




$\begin{array}{lllll}\text { C7 } & 0.3493(3) & 0.55934(8) & 0.3385(2) & 0.0511(4) \\ \text { H7 } & 0.4862 & 0.5602 & 0.3778 & 0.061^{*} \\ \text { C8 } & 0.2464(3) & 0.61115(7) & 0.2972(2) & 0.0493(4) \\ \text { H8 } & 0.3113 & 0.6478 & 0.3096 & 0.059^{*} \\ \text { C9 } & 0.0444(3) & 0.60757(7) & 0.2367(3) & 0.0490(4) \\ \text { H9 } & -0.0242 & 0.6428 & 0.2078 & 0.059^{*} \\ \text { C10 } & 0.2527(2) & 0.30146(7) & 0.4321(2) & 0.0380(3) \\ \text { H10A } & 0.3269 & 0.3242 & 0.3463 & 0.046^{*} \\ \text { H10B } & 0.2369 & 0.3259 & 0.5380 & 0.046^{*} \\ \text { C11 } & 0.3674(2) & 0.24555(7) & 0.4832(2) & 0.0365(3) \\ \text { N1 } & 0.01710(17) & 0.39939(5) & 0.28821(16) & 0.0340(3) \\ \text { N2 } & -0.27844(18) & 0.34541(6) & 0.18112(18) & 0.0391(3) \\ \text { N3 } & -0.0580(2) & 0.55666(6) & 0.21748(19) & 0.0432(3) \\ \text { O1 } & 0.30963(19) & 0.19622(5) & 0.4458(2) & 0.0623(4) \\ \text { O2 } & 0.53762(17) & 0.25707(5) & 0.57657(19) & 0.0544(3) \\ \text { H2 } & 0.5945 & 0.2257 & 0.6057 & 0.082^{*} \\ \text { S1 } & 0.00702(6) & 0.280700(17) & 0.33349(6) & 0.04496(15)\end{array}$

Atomic displacement parameters $\left(\AA^{2}\right)$

\begin{tabular}{lllllll}
\hline & $U^{11}$ & $U^{22}$ & $U^{33}$ & $U^{12}$ & $U^{13}$ & $U^{23}$ \\
\hline C1 & $0.0331(7)$ & $0.0315(7)$ & $0.0371(8)$ & $-0.0008(6)$ & $-0.0044(6)$ & $0.0020(6)$ \\
C2 & $0.0329(7)$ & $0.0395(8)$ & $0.0500(9)$ & $0.0000(6)$ & $-0.0100(6)$ & $0.0044(7)$ \\
C3 & $0.0357(7)$ & $0.0329(8)$ & $0.0477(9)$ & $0.0046(6)$ & $-0.0053(6)$ & $0.0035(6)$ \\
C4 & $0.0344(7)$ & $0.0297(7)$ & $0.0338(7)$ & $0.0004(5)$ & $0.0004(6)$ & $0.0001(5)$ \\
C5 & $0.0373(7)$ & $0.0279(7)$ & $0.0345(7)$ & $0.0011(6)$ & $-0.0004(6)$ & $-0.0005(6)$ \\
C6 & $0.0425(8)$ & $0.0330(8)$ & $0.0512(9)$ & $-0.0010(6)$ & $-0.0106(7)$ & $0.0027(7)$ \\
C7 & $0.0470(9)$ & $0.0454(10)$ & $0.0591(11)$ & $-0.0091(7)$ & $-0.0137(8)$ & $-0.0012(8)$ \\
C8 & $0.0612(10)$ & $0.0321(8)$ & $0.0543(10)$ & $-0.0104(7)$ & $0.0000(8)$ & $-0.0057(7)$ \\
C9 & $0.0564(10)$ & $0.0281(8)$ & $0.0628(11)$ & $0.0042(7)$ & $0.0045(8)$ & $-0.0008(7)$ \\
C10 & $0.0340(7)$ & $0.0297(8)$ & $0.0491(9)$ & $0.0002(6)$ & $-0.0078(6)$ & $0.0004(6)$ \\
C11 & $0.0324(7)$ & $0.0313(8)$ & $0.0452(8)$ & $0.0003(6)$ & $-0.0041(6)$ & $-0.0005(6)$ \\
N1 & $0.0328(6)$ & $0.0284(6)$ & $0.0399(7)$ & $-0.0004(5)$ & $-0.0052(5)$ & $0.0024(5)$ \\
N2 & $0.0347(6)$ & $0.0331(7)$ & $0.0482(7)$ & $-0.0028(5)$ & $-0.0096(5)$ & $0.0040(5)$ \\
N3 & $0.0418(7)$ & $0.0286(7)$ & $0.0587(8)$ & $0.0041(5)$ & $0.0000(6)$ & $0.0007(6)$ \\
O1 & $0.0526(7)$ & $0.0291(6)$ & $0.1011(11)$ & $-0.0001(5)$ & $-0.0329(7)$ & $-0.0024(6)$ \\
O2 & $0.0424(6)$ & $0.0322(6)$ & $0.0852(9)$ & $0.0028(5)$ & $-0.0269(6)$ & $-0.0038(6)$ \\
S1 & $0.0384(2)$ & $0.0272(2)$ & $0.0670(3)$ & $-0.00354(15)$ & $-0.01691(18)$ & $0.00762(17)$ \\
& & & & & &
\end{tabular}

Geometric parameters $\left(\AA,{ }^{\circ}\right)$

\begin{tabular}{llll}
\hline $\mathrm{C} 1-\mathrm{N} 1$ & $1.3276(18)$ & $\mathrm{C} 7-\mathrm{C} 8$ & $1.372(2)$ \\
$\mathrm{C} 1-\mathrm{N} 2$ & $1.3466(18)$ & $\mathrm{C} 7-\mathrm{H} 7$ & 0.9300 \\
$\mathrm{C} 1-\mathrm{S} 1$ & $1.7507(15)$ & $\mathrm{C} 8-\mathrm{C} 9$ & $1.380(2)$ \\
$\mathrm{C} 2-\mathrm{N} 2$ & $1.3377(19)$ & $\mathrm{C} 8-\mathrm{H} 8$ & 0.9300 \\
$\mathrm{C} 2-\mathrm{C} 3$ & $1.369(2)$ & $\mathrm{C} 9-\mathrm{N} 3$ & $1.331(2)$ \\
$\mathrm{C} 2-\mathrm{H} 2 \mathrm{~A}$ & 0.9300 & $\mathrm{C} 9-\mathrm{H} 9$ & 0.9300 \\
$\mathrm{C} 3-\mathrm{C} 4$ & $1.3909(19)$ & $\mathrm{C} 10-\mathrm{C} 11$ & $1.503(2)$
\end{tabular}




\begin{tabular}{|c|c|c|c|}
\hline $\mathrm{C} 3-\mathrm{H} 3$ & 0.9300 & $\mathrm{C} 10-\mathrm{S} 1$ & $1.7964(14)$ \\
\hline $\mathrm{C} 4-\mathrm{N} 1$ & $1.3453(17)$ & $\mathrm{C} 10-\mathrm{H} 10 \mathrm{~A}$ & 0.9700 \\
\hline $\mathrm{C} 4-\mathrm{C} 5$ & $1.4815(19)$ & $\mathrm{C} 10-\mathrm{H} 10 \mathrm{~B}$ & 0.9700 \\
\hline $\mathrm{C} 5-\mathrm{N} 3$ & $1.3401(18)$ & $\mathrm{C} 11-\mathrm{O} 1$ & $1.1991(18)$ \\
\hline $\mathrm{C} 5-\mathrm{C} 6$ & $1.385(2)$ & $\mathrm{C} 11-\mathrm{O} 2$ & $1.3085(17)$ \\
\hline $\mathrm{C} 6-\mathrm{C} 7$ & $1.381(2)$ & $\mathrm{O} 2-\mathrm{H} 2$ & 0.8200 \\
\hline $\mathrm{C} 6-\mathrm{H} 6$ & 0.9300 & & \\
\hline $\mathrm{N} 1-\mathrm{C} 1-\mathrm{N} 2$ & $126.48(13)$ & $\mathrm{C} 7-\mathrm{C} 8-\mathrm{C} 9$ & $118.45(15)$ \\
\hline $\mathrm{N} 1-\mathrm{C} 1-\mathrm{S} 1$ & $121.11(10)$ & $\mathrm{C} 7-\mathrm{C} 8-\mathrm{H} 8$ & 120.8 \\
\hline $\mathrm{N} 2-\mathrm{C} 1-\mathrm{S} 1$ & $112.41(10)$ & $\mathrm{C} 9-\mathrm{C} 8-\mathrm{H} 8$ & 120.8 \\
\hline $\mathrm{N} 2-\mathrm{C} 2-\mathrm{C} 3$ & $122.31(13)$ & $\mathrm{N} 3-\mathrm{C} 9-\mathrm{C} 8$ & $123.84(15)$ \\
\hline $\mathrm{N} 2-\mathrm{C} 2-\mathrm{H} 2 \mathrm{~A}$ & 118.8 & $\mathrm{~N} 3-\mathrm{C} 9-\mathrm{H} 9$ & 118.1 \\
\hline $\mathrm{C} 3-\mathrm{C} 2-\mathrm{H} 2 \mathrm{~A}$ & 118.8 & $\mathrm{C} 8-\mathrm{C} 9-\mathrm{H} 9$ & 118.1 \\
\hline $\mathrm{C} 2-\mathrm{C} 3-\mathrm{C} 4$ & $117.60(13)$ & $\mathrm{C} 11-\mathrm{C} 10-\mathrm{S} 1$ & $108.22(10)$ \\
\hline $\mathrm{C} 2-\mathrm{C} 3-\mathrm{H} 3$ & 121.2 & $\mathrm{C} 11-\mathrm{C} 10-\mathrm{H} 10 \mathrm{~A}$ & 110.1 \\
\hline $\mathrm{C} 4-\mathrm{C} 3-\mathrm{H} 3$ & 121.2 & $\mathrm{~S} 1-\mathrm{C} 10-\mathrm{H} 10 \mathrm{~A}$ & 110.1 \\
\hline $\mathrm{N} 1-\mathrm{C} 4-\mathrm{C} 3$ & $121.20(13)$ & $\mathrm{C} 11-\mathrm{C} 10-\mathrm{H} 10 \mathrm{~B}$ & 110.1 \\
\hline $\mathrm{N} 1-\mathrm{C} 4-\mathrm{C} 5$ & $117.57(12)$ & $\mathrm{S} 1-\mathrm{C} 10-\mathrm{H} 10 \mathrm{~B}$ & 110.1 \\
\hline $\mathrm{C} 3-\mathrm{C} 4-\mathrm{C} 5$ & $121.23(13)$ & $\mathrm{H} 10 \mathrm{~A}-\mathrm{C} 10-\mathrm{H} 10 \mathrm{~B}$ & 108.4 \\
\hline $\mathrm{N} 3-\mathrm{C} 5-\mathrm{C} 6$ & $122.60(14)$ & $\mathrm{O} 1-\mathrm{C} 11-\mathrm{O} 2$ & $123.76(14)$ \\
\hline $\mathrm{N} 3-\mathrm{C} 5-\mathrm{C} 4$ & $115.89(12)$ & $\mathrm{O} 1-\mathrm{C} 11-\mathrm{C} 10$ & $124.47(13)$ \\
\hline $\mathrm{C} 6-\mathrm{C} 5-\mathrm{C} 4$ & $121.49(13)$ & $\mathrm{O} 2-\mathrm{C} 11-\mathrm{C} 10$ & $111.76(12)$ \\
\hline $\mathrm{C} 7-\mathrm{C} 6-\mathrm{C} 5$ & $118.94(15)$ & $\mathrm{C} 1-\mathrm{N} 1-\mathrm{C} 4$ & $116.48(12)$ \\
\hline $\mathrm{C} 7-\mathrm{C} 6-\mathrm{H} 6$ & 120.5 & $\mathrm{C} 2-\mathrm{N} 2-\mathrm{C} 1$ & $115.86(12)$ \\
\hline $\mathrm{C} 5-\mathrm{C} 6-\mathrm{H} 6$ & 120.5 & $\mathrm{C} 9-\mathrm{N} 3-\mathrm{C} 5$ & $117.29(14)$ \\
\hline $\mathrm{C} 8-\mathrm{C} 7-\mathrm{C} 6$ & $118.87(15)$ & $\mathrm{C} 11-\mathrm{O} 2-\mathrm{H} 2$ & 109.5 \\
\hline $\mathrm{C} 8-\mathrm{C} 7-\mathrm{H} 7$ & 120.6 & $\mathrm{C} 1-\mathrm{S} 1-\mathrm{C} 10$ & $101.46(7)$ \\
\hline $\mathrm{C} 6-\mathrm{C} 7-\mathrm{H} 7$ & 120.6 & & \\
\hline
\end{tabular}

Hydrogen-bond geometry $\left(\AA,{ }^{\circ}\right)$

\begin{tabular}{lllll}
\hline$D-\mathrm{H} \cdots A$ & $D-\mathrm{H}$ & $\mathrm{H} \cdots A$ & $D \cdots A$ & $D-\mathrm{H} \cdots A$ \\
\hline $\mathrm{O} 2-\mathrm{H} 2 \cdots \mathrm{N} 2^{\mathrm{i}}$ & 0.82 & 1.87 & $2.694(2)$ & 178 \\
$\mathrm{C} 2-\mathrm{H} 2 A \cdots \mathrm{O} 1^{\mathrm{ii}}$ & 0.93 & 2.58 & $3.230(2)$ & 127 \\
$\mathrm{C} 8-\mathrm{H} 8 \cdots \mathrm{O} 2^{\mathrm{iii}}$ & 0.93 & 2.48 & $3.392(2)$ & 165 \\
$\mathrm{C} 9-\mathrm{H} 9 \cdots{ }^{\mathrm{ii}}$ & 0.93 & 2.45 & $3.296(2)$ & 151 \\
\hline
\end{tabular}

Symmetry codes: (i) $x+1,-y+1 / 2, z+1 / 2$; (ii) $x-1,-y+1 / 2, z-1 / 2$; (iii) $-x+1,-y+1,-z+1$; (iv) $-x, y+1 / 2,-z+1 / 2$. 